\section{Editor's introduction: Minimum TOS understanding: heterogeneity vs sampling procedures}

Kim H. Esbensen

andependent researcher, consultant, owner, KHE Consulting, Copenhagen

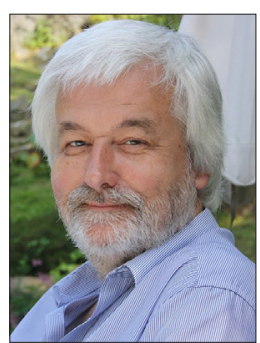

In order to be reliable, business decisions must be based on reliable analytical results, which in turn must be based on representative samples from the materials, lots and process streams. Thus, in one sense everything starts with being able to conduct appropriate sampling of all types of materials and lots in academe, technology, industry, trade, commerce and society. "Appropriate sampling" means "representative sampling". Otherwise, "What is the meaning of analysing a sample that cannot be documented to be representative? None, there is no meaning-it is only a waste of money." As it turns out, representative sampling is only dependent on two critical success factors: i) how to counteract the debilitating influence on sampling from material heterogeneity and ii) only using composite samplingnever grab sampling. It is as simple as that...

In a few more words:

Sampling procedures and equipment must be able to counteract the

\section{DOI: $10.1255 /$ sew.2021.a31}

(c) 2021 The Author

Published under a Creative Commons BY-NC-ND licence

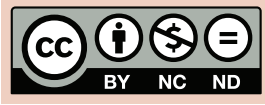

vastly different degrees of heterogeneity encountered in all materials and lots (stationary or moving) in need of reliable compositional characterisation. Business leaders must acknowledge, and understand, heterogeneity.

Sampling procedures must be representative, i.e. bias-free. Of the two most common sampling approaches used today, one is demonstrably not so-grab sampling. Only composite sampling can be made fit-for-purpose representative for all materials, at all scales and under all sampling conditions. Business leaders must understand this and decree only to use composite sampling.

This is really all there is to it...

By investing the miniscule effort needed to understand the above, management will actually have fulfilled its role; the rest can be left to the technical operative levels, but it is of course unsatisfactory to lead if not reasonably well informed about what, when and how the raw materials and the processes involved bring about the final product.

Here follows the minimum TOS knowledge needed at all levels-it isn't much. The first issue is often highly surprising, but it opens up for the singular critical insight needed:

Sampling of materials, processes a.o. targets for which reliable analytical results are needed is a process that is not quantitively reproducible, i.e. repeated sampling (two, three or more "control samplings for example" will give rise to different analytical results. There is always a larger or smaller sampling variability (call it sampling spread if this is clearer for the reader). Why is that?

Because all materials and processes in technology, industry and society are heterogeneous.

Because grab sampling is fundamentally unable to counteract the intrinsic heterogeneity met with in all materials, lots and processes for which business decisions have to be made, the results will be an undesirably broad spread of analytical results. The unacceptable consequences of a too-broad sampling + analysis spread is laid out in full below.

One would always wish for low material heterogeneity, but it is seldom possible to alter this for original lots easily without significant, and almost always prohibitive, economic costs. Is there another way? Yes, composite sampling.

Thus, both an ill-informed sampling approach (grab sampling) and/or sampling significantly heterogeneous materials, lots and processes without proper amelioration (grab sampling) will always result in a seriously inflated sampling + analysis spread.

Enter the TOS, the world's only fully comprehensive framework for 


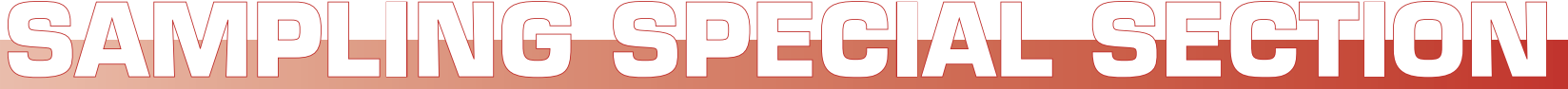
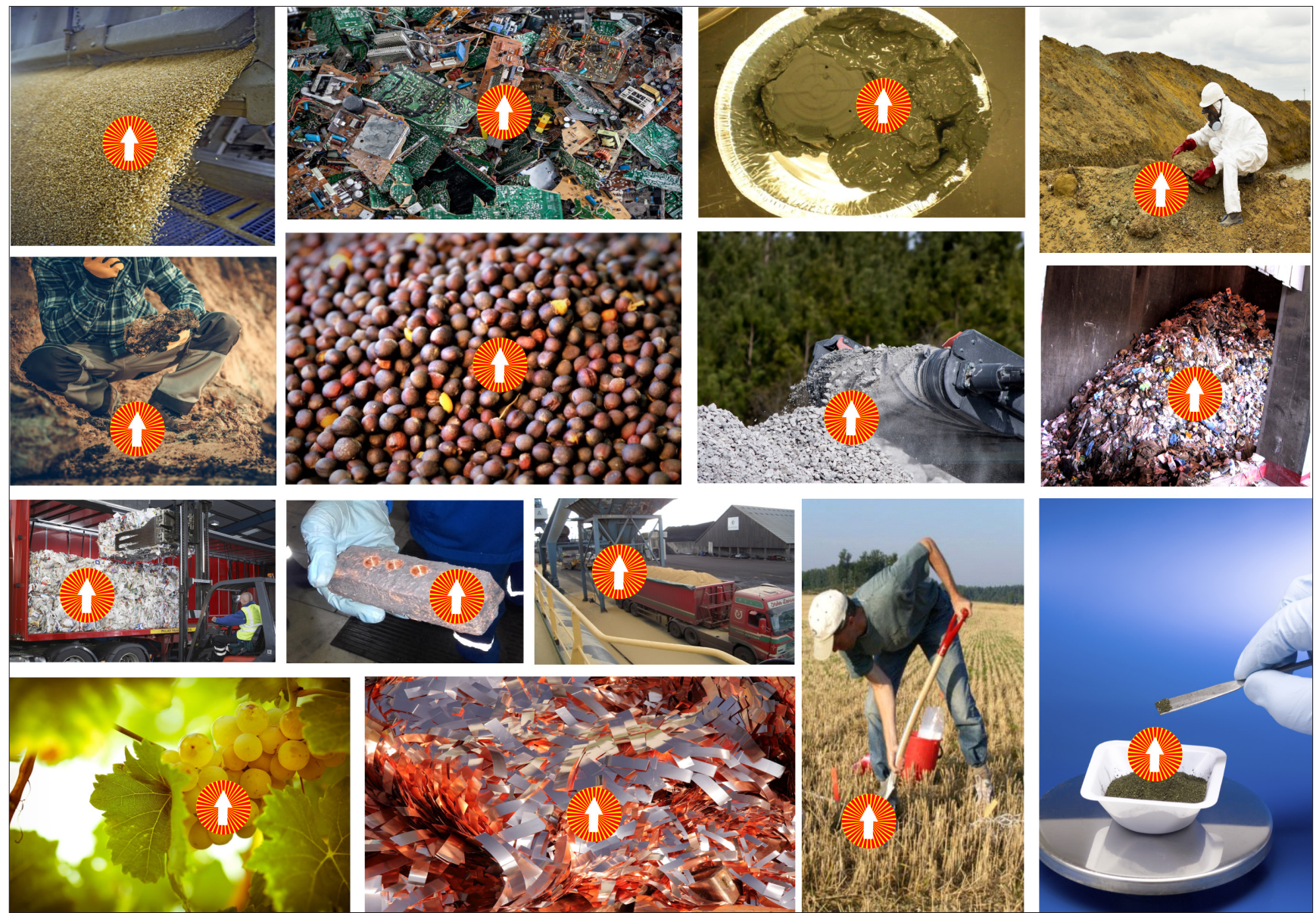

Figure A. Heterogeneous materials, lots, processes are legion and come in a plethora of forms, containers, vessels etc. Because of heterogeneity, there will always be a significant sampling variability (sampling spread). Representativity w.r.t. the whole lot demands aggregating an appropriate number of increments covering the entire lot volume. It is clear why singular grab samples will always result in different analytical results, since they are extracted from different spatial locations. Repeated grab sampling will produce a larger or smaller sampling + analysis spread. Composite samples must contain a material-dependent necessary-and-sufficient number of increments in order to secure a "fit-for-purpose" representativity status. Composite sampling will also lead to a non-vanishing sampling + analysis spread, but with a much reduced magnitude, see Figure D. The TOS is the world's only necessary-and-sufficient framework for counteracting heterogeneity in the most effective way, always leading to a minimised effective sampling spread.

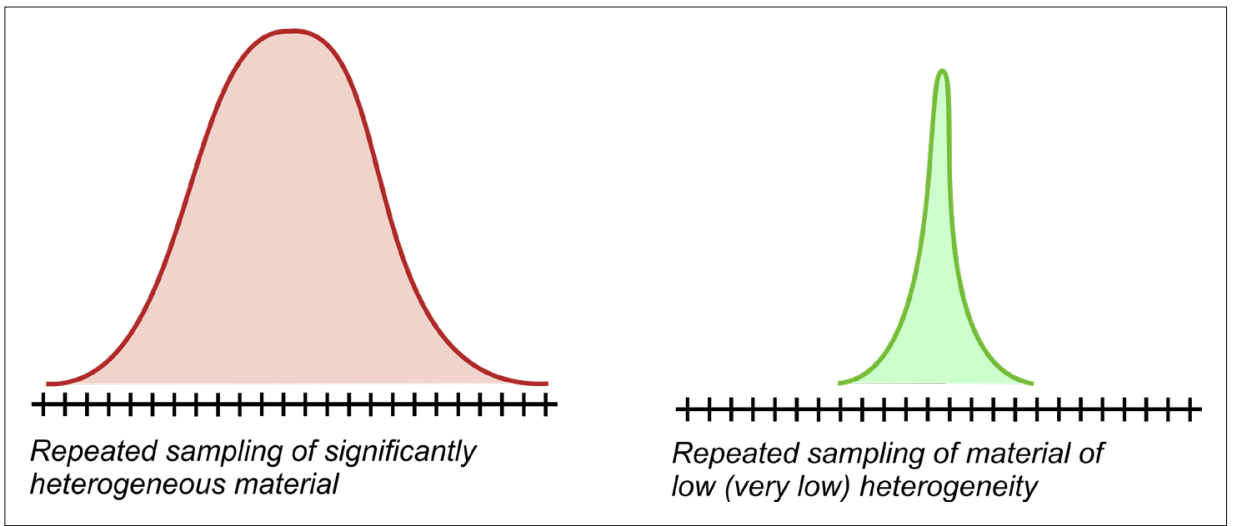

Figure B. Grab sampling of materials with widely differing heterogeneity will result in a characteristic sampling + analysis spread, the width of which is a direct reflection of the magnitude of the heterogeneity. The unacceptable business consequences of a too-broad sampling + analysis spread is laid out in full below. 


\section{SAMULWNG SPEGHL SEGHON}

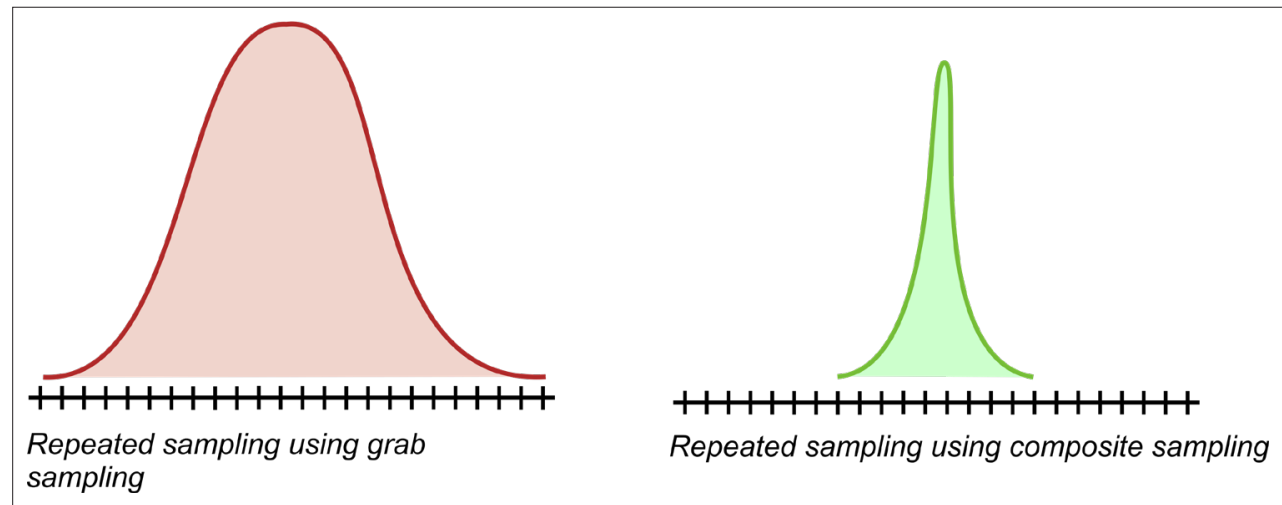

Figure C. Sampling spread as a function of using a non-representative procedure (grab sampling) compared to the world's only fully representative approach, composite sampling. The unacceptable business consequences of a too-broad sampling + analysis spread is laid out in full below.

representative sampling. The TOS stipulates why and how to use composite sampling for all materials regardless of their level of heterogeneity, Figures A and B. The TOS also outlines how to calibrate composite sampling (determination of the necessary-and-sufficient number of increments to aggregate) to be able to counteract heterogeneity at whatever level encountered (low, intermediate, high). The TOS is the world's only directive for how to implement representative sampling solutions that eliminate the negative effects from the two key critical success factors: heterogeneity and choice of sampling procedure.

\section{Business decision}

consequences of not involving the TOS

For the reasons laid out above there is always an inherent, non-zero risk of making decisions based on inferior or downright wrong information, in this case numerical information (analytical results) which are fraught with unnecessary sampling + analysis uncertainty. Risk management is a due diligence requirement at the business level. With the few fundamentals laid bare above, risk management must include a minimum topical understanding of the risks stemming from sampling vs heterogeneity issues which all take place before analysis.

Making sure of optimal analytical performance is not enough-because the quality of analytical results depends much more on the preceding quality of the sampling procedures employed. Sampling uncertainties are typically 5-10-25 times larger than the optimised analytical laboratory performance-in direct proportionality to how well the sampling procedure has succeeded in mitigating the detrimental influence from heterogeneity-or not.

Inside or outside the analytical laboratory - that is the question!

Scores of examples exist of futile expansion of analytical departments with next to no additional gain in the form of improved business decision making. While knowledge and experiences with the entities behind such examples are obviously highly confidential, what can be revealed is that behind every known example there are equally many records of successful make-over operations-which all involved introduction of proper TOS knowledge to the corporation, company or organisation involved. It is difficult to put exact numbers on the economic gains (or thwarted losses) in these examples, but a start would be: What are the costs for a new analytical lab? For a significantly upgraded laboratory? For hiring one or more scientists or technicians? Compare this to now knowing for a fact that the root cause for particular bottom-line issues lies outside the laboratory! Enter the TOS, with which to clear up any-andall sampling deficits-these alternative

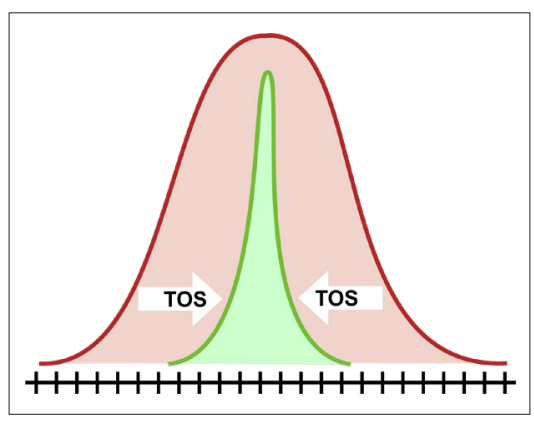

Figure D. The The solution guaranteeing representative sampling of significantly heterogeneous materials is always using appropriate sampling procedures (composite sampling)-the TOS.

costs will in most cases have difficulty reaching even a fraction of what would have been waster on the "laboratory expansion" avenue.

Resolving such issues lies at the heart of successful risk management at the top management level. The alternative, being ignorant of the consequences of not caring about the mere "technicality" of sampling, is an assured inferior bottom line result without anyone in the organisation being able to point to viable remediation avenues... the TOS to the fore!

To motivate readers to include a smattering of the TOS in the risk management setup of their operation is the very thrust of this Sampling Column. Below we present a bonanza of economic arguments for involving the TOS at all levels. 